\title{
On cyclic tournaments
}

\author{
Noboru ITO \\ (Received January 30, 1991)
}

1.

Let $V$ be the set of integers $1,2, \ldots, v$ and $S(v)$ the symmetric group on $V$. Put $C=(1,2, \ldots, v)$. Let $W(v)$ be the set of all subgroups of $S(v)$ of odd orders containing $C$.

A complete asymmetric digraph $A$ whose set of vertices is $V$ is also called a tournament. We identify a digraph with its adjacency matrix. We also identify a permutation with its matrix representation.

Let $A$ and $B$ be two tournaments of order $v$. Then $B$ is equivalent to $A$ if there exists a permutation matrix $P$ such that $B=P^{t} A P$, where $t$ denotes the transposition. This is a true equivalence relation. If $B=A$, then $P$ is called an automorphism of $A$. The set $G(A)$ of all automorphisms of $A$ forms a group, the automorphism group of $A$.

A tournament $A$ is called cyclic if $G(A)$ contains $C$. Let $A$ be a cyclic tournament of order $v$. We may regard the first row vector $O(1)$ of $A$ as the out-neighborhood of the vertex 1 . Since $A$ is cyclic, $A$ is completely determined by $O(1)$. Put $v=2 k+1$ and $i^{*}=v-i+1$ for $2 \leqq i \leqq k$ +1 . We call $\left\{i, i^{*}\right\}$ a complementary pair for $2 \leqq i \leqq k+1$. Choose one element from each complementary pair. This procedure determines $O(1)$ and hence $A$. Thus there exist $2^{k}$ cyclic tournaments. Let $C(v)$ be the set of all cyclic tournaments of order $v$.

Let $G$ be an element of $W(v)$ and $H$ the stabilizer of 1 in $G$. If we want to construct a cyclic tournament $A$ such that $G(A)$ contains $G$, then we have a restriction on the choice of elements from complementary pairs imposed by $H$. Namely if $i$ and $j^{*}$ belong to the same orbit of $H$, then both of $i$ and $j^{*}$ or none of them have to be chosen. If we do so, then we see that every maximal element $G$ of $W(v)$ is of the form $G=G(A)$ for some element $A$ of $C(v)$.

Let $v=p$ be a prime and $u(p)$ the odd portion of $p-1$, namely $p-1=$ $2^{e} u(p)$. Then $G(p)$ denotes the metacyclic group of order $p u(p)$ on $V$. Since $G(p)$ is maximal in $W(p), G(p)=G(A)$ for some tournament $A$ of order $p$.

In the present paper we show the following : 
(i) If any $v$-cycle of $G(A)$ is a power of $C$, we can determine the size of the equivalence class of $A$ in $C(v)$;

(ii) Any maximal element of $W(v)$ is of the form $G\left(p_{1}\right)^{\circ} G\left(p_{2}\right)^{\circ} \ldots$ ${ }^{\circ} G\left(p_{r}\right)$, where $v=p_{1} p_{2} \ldots p_{r}$ is a prime decomposition of $v$ and ${ }^{\circ}$ denotes the Polya composition. For this see [1];

(iii) An element of $W(v)$ of the largest order is uniquely (up to the conjugacy in $W(v)$ ) determined by a certain linear order of odd primes ;

and

(iv) Any element of $W(v)$ is of the form $G(A)$ for a certain element $A$ of $C(v)$.

2.

Proposition 1. Let $A$ and $B$ be two equivalent cyclic tournaments such that $B=P^{t} A P$, where $P$ is a permutation matrix. Assume that any $v$-cycle of $G(A)$ is a power of $C$. Then $P$ belongs to the normalizer $N(\langle C\rangle)$ of $\langle C\rangle$. Put $N(\langle C\rangle)=\langle C\rangle N(v)$, where $N(v)$ is the stabilizer of 1 in $N(\langle C\rangle)$. Then $N(v)$ is Abelian of order $\varphi(v)$, where $\varphi$ denotes the Euler totient function. Let $\varphi(A)$ be the order of $N(v) \cap G(A)$. Then the size of the equivalence class in $C(v)$ to which $A$ belongs equals $\varphi(v) / \varphi(A)$.

Proof. We have that $A=P B P^{t}=P C^{t} B C P^{t}=P C^{t} P^{t} A P C P^{t}$. So $P C P^{t}$ belongs to $G(A)$. By assumption $P C P^{t}$ is a power of $C$. The rest is obvious.

REMARK 1. The assumption on $G(A)$ in proposition 1 is satisfied, in particular, if $G(A)=\langle C\rangle$ or $v$ is square-free. So for certain $v$ it is possible to have a formula for the number of equivalence classes of cyclic tournaments.

(i) If $v$ is a Fermat prime, $v=2^{m}+1$, then each equivalence class has size $v-1$ and hence there exist $2^{2^{m-1-m}}$ classes.

(ii) If $v$ and $(v-1) / 2=k$ are primes, then, since any tournament $A$ of order $v$ such that $G(A)$ has order $v k$ is equivalent to the tournament of quadratic residue (or non-residue) type ([2]), there exist $\left(2^{k-1}-1 / k\right)+1$ classes.

3.

Proposition 2. Let $G$ be a maximal element of $W(v)$. Then $G$ is similar to $G\left(p_{1}\right)^{\circ} G\left(p_{2}\right)^{\circ} \ldots{ }^{\circ} G\left(p_{r}\right)$, where ${ }^{\circ}$ denotes the Polya composition and $v=p_{1} p_{2} \ldots p_{r}$ is a prime decomposition.

PROOF. If $v$ is a prime, then our assertion holds good by a theorem 
of Burnside ([4], (11.7)). So assume that $v$ is not a prime. Since $G$ contains $C$, by a theorem of Schur $([4],(25.3)) G$ is imprimitive. Let $M$ be a maximal subgroup of $G$ of index $m$ containing $G_{1}$, the stabilizer of 1 in $G$. Let core $(M)$ denote the largest normal subgroup of $G$ contained in $M$. Then $G / \operatorname{core}(M)$ is a permutation group of degree $m$ and of odd order containing an $m$-cycle. Since $M$ is maximal in $G$, by a theorem of Schur ([4], (25.3)) we have that $m$ is a prime. Now we apply an induction argument with respect to the degree. For the rest we refer to ([3], 10.5.5).

REMARK 2. We notice that, under the assumption that $G$ contains a $v$-cycle, we have shown the solvability of $G$ without invoking the FeitThompson theorem.

4.

Let $P$ be the set of all odd primes. We introduce a new order in $P$ as follows : $p \gg q$ if and only if $(q u(q))^{p-1}>(p u(p))^{q-1}$.

LEMMA $1 . \quad \gg$ is a linear order.

PROOF. If $p \neq q$, then $(q u(q))^{p-1} \neq(p u(p))^{q-1}$. Now assume that $p \gg q$ and $q \gg r$. Then we have that $(q u(q))^{p-1}>(p u(p))^{q-1}$ and $(r u(r))^{q-1}>$ $(q u(q))^{r-1}$. So it follows that $(q u(q))^{(p-1)(r-1)}>(p u(p))^{(q-1)(r-1)}$ and that $(r u(r))^{(q-1)(p-1)}>(q u(q))^{(r-1)(p-1)}$. Hence we have that $(r u(r))^{p-1}>$ $(p u(p))^{r-1}$, namely $p \gg r$.

REMARK 3. The following is the sequence of odd primes under 100 in the increasing order using $\gg: 3,7,5,11,13,19,23,31,29,17,43,37,47,41$, $59,67,61,71,79,83,73,89,97$.

PROPOSITION 3. Let $G$ be an element of $W(v)$ of the largest order. Then $G=G\left(p_{1}\right)^{\circ} G\left(p^{2}\right)^{\circ} \ldots{ }^{\circ} G\left(p_{r}\right)$, where $v=p_{1} p_{2} \ldots p_{r}$ is a prime decomposition such that $p_{1} \geqq p_{2} \geqq \ldots \geqq p_{\text {r. }}$

Proof. The case $r=1$ is trivial. Assume that $r=2$. Then the orders of $G\left(p_{1}\right)^{\circ} G\left(p_{2}\right)$ and $G\left(p_{2}\right)^{\circ} G\left(p_{1}\right)$ are equal to $p_{1} u\left(p_{1}\right)\left(p_{2} u\left(p_{2}\right)\right)^{p_{1}}$ and $p_{2} u\left(p_{2}\right)\left(p_{1} u\left(p_{1}\right)\right)^{p_{2}}$ respectively. So if $p_{1} \gg p_{2}$, then the order of $G\left(p_{1}\right)^{\circ} G\left(p_{2}\right)$ is larger than that of $G\left(p_{2}\right)^{\circ} G\left(p_{1}\right)$. Now assume that $r \geqq 3$ and put $v=$ $p_{1} p_{2} z$. Then by an induction argument on $r$ it is sufficient to compare the orders of $G_{1}=G\left(p_{1}\right)^{\circ} G\left(p_{2}\right)^{\circ} G(z)$ and $G_{2}=G\left(p_{2}\right)^{\circ} G\left(p_{1}\right)^{\circ} G(z)$, where $G(z)=$ $G\left(p_{3}\right)^{\circ} \ldots{ }^{\circ} G\left(p_{r}\right)$. In particular, we may assume that $p_{1} \neq p_{2}$. Let $g(z)$ denote the order of $G(z)$. Now the orders of $G_{1}$ and $G_{2}$ are equal to $p_{1} u\left(p_{1}\right)\left(p_{2} u\left(p_{2}\right) g(z)^{p_{2}}\right)^{p_{1}}$ and $p_{2} u\left(p_{2}\right)\left(p_{1} u\left(p_{1}\right) g(z)^{p_{1}}\right)^{p_{2}}$ respectively. So exact- 
ly as in the case where $r=2$, we see that the order of $G_{1}$ is larger than that of $G_{2}$.

REMARK 4. Though $G$ is unique up to the conjugacy in $S(v)$, there may exist many inequivalent $A$ 's such that $G(A)=G$.

5 .

Let $O(i)$ denote the out-neighborhood of $i, 1 \leqq i \leqq v$.

LEMMA 2. Let $A$ be a cyclic tournament of order $v$ such that the out-neighborhood $O(1)$ of the vertex 1 consists of $2,3, \ldots, k$, where $v=2 k$ +1 . Then $G(A)=\langle C\rangle$.

PROOF. It is enough to notice that $O(1) \cap O(i)$ contains $k-i+1$ vertices for $1 \leqq i \leqq k+1$, which implies that the stabilizer of 1 in $G(A)$ is trivial.

REMARK 5. We remark that we have $G(A)=\langle C\rangle$ for most cyclic tournaments $A$.

LEMMA 3. Let $X$ and $Y$ be elements of $W(v)$ such that $X$ contains $Y$ properly. Let $X(1)$ and $Y(1)$ be the stabilizers of 1 in $X$ and $Y$ respectively. Then $X(1)$ and $Y(1)$ have distinct orbit decompositions on $V-\{1\}$.

PROOF. We apply an induction argument on the order $v$. If $v$ is a prime, then, by a theorem of Burnside [4,11.7], $X(1)$ and $Y(1)$ are semiregular on $V-\{1\}$ and $X(1)$ contains $Y(1)$ properly. So the assertion is obvious. If $v$ is not a prime, then, by a theorem of Schur $[4,25.3] X$ is imprimitive. Let $D$ be a non-trivial block and $X(D)$ and $Y(D)$ the global stabilizers of $D$ in $X$ and $Y$ respectively. Since $Y$ contains $C$, $Y(D)$ is transitive on $D . \quad X(1)$ and $Y(1)$ are the stabilizers of 1 in $X(D)$ and $Y(D)$ respectively. Then by induction hypothesis the orbit decomposition of $Y(1)$ is a proper refinement of that of $X(1)$ on $D-\{1\}$.

Proposition 4. Let $W$ be an element of $W(v)$. Then there exists a cyclic tournament $A$ of order $v$ such that $W=G(A)$.

PROOF. In $\S 1$ we described a procedure to construct a cyclic tournament $A^{\circ}$ such that $G\left(A^{\circ}\right)$ contains $W$. Now assume that $G\left(A^{\circ}\right)$ contains $W$ properly. Let $G\left(A^{\circ}\right)(1)$ and $W(1)$ be the stabilizers of 1 in $G\left(A^{\circ}\right)$ and $W$ respectively. Then by Lemma 3 the orbit decomposition of $V-\{1\}$ by $W(1)$ is a proper refinement of that by $G\left(A^{\circ}\right)(1)$. So by the procedure 
described in $\S 1$ we can construct a cyclic tournament $A^{\circ \circ}$ such that $G\left(A^{\circ \circ}\right)$ contains $W$ and $G\left(A^{\circ}\right)$ contains $G\left(A^{\circ \circ}\right)$ properly. We may repeat this process. So eventually we obtain a cyclic tournament $A$ such that $G(A)=W$.

\section{References}

[1] M. Goldberg and J. W. Moon, On the composition of two tournaments. Duke Math. J. 37 (1970), 323-332.

[2] E. C. JOHNSEN, Skew-Hadamard Abelian group difference sets. J. Algebra 4 (1966), 388-402.

[ 3 ] W. R. ScOTT, Group Theory. Prentice-Hall, 1964.

[4] H. WiElandT, Finite permutation groups. Academic Press, 1964.

Department of Mathematics Meijo University

Nagoya-Tenpaku, Japan 468 